\title{
Combination Therapy Using Adipose-Derived Stem Cells And Low-Intensity Extracorporeal Shockwave Therapy On Erectile Dysfunction In Streptozotocin- Induced Diabetic Rats
}

\section{Dankang Li}

Zhengzhou University

\section{Yongchao Ge}

Zhengzhou Third People's Hospital

Quanliang Hou

Zhengzhou University

\section{Wenhua Wang}

Zhengzhou University

Chengda Zhang

Beaumont Health

\section{Mengying Ge}

Zhengzhou University

Huizi Tian

Zhengzhou University

Qinglin Zhao

Zhengzhou University

\section{Opolot Godfrey}

Zhengzhou University

Weidong Zhang ( $D$ imooni@163.com )

Zhengzhou University

\section{Research article}

Keywords: adipose-derived stem cells, low-intensity extracorporeal shockwave therapy, erectile dysfunction, diabetes mellitus, rat model

Posted Date: August 23rd, 2019

DOI: https://doi.org/10.21203/rs.2.13432/v1

License: (c) (i) This work is licensed under a Creative Commons Attribution 4.0 International License. Read Full License 
Page $2 / 25$ 


\section{Abstract}

Background $\triangle$ Diabetes mellitus (DM) is an important risk factor for erectile dysfunction (ED). Adipose-derived stem cells (ADSCs) and low-intensity extracorporeal shockwave therapy (LI-ESWT) are two emerging therapies for ED. The efficacy of ADSCs combined LI-ESWT in the treatment of diabetes induced erectile dysfunction (DMED) was not completely elucidated. This study aim to investigate combined therapeutic efficacy of ADSCs and LI-ESWT in rat model of DMEDand whether they have interaction.

Methods $\varangle$ Rats were randomly divided into 5 groups: control, erectile dysfunction group (ED), ADSCs treatment group(ADSCs), LI-ESWT treatment group (LI-ESWT) and ADSCs combined with LI-ESWT treatment (ADSCs+LI-ESWT). After 4 weeks, erectile function was assessed using intracavernous pressure (ICP) and mean arterial pressure (MAP), after that performed immunohistochemistry $(\mathrm{IH})$.

Results囚Finally, a total of 98 rats completed the experiment. The ICP/MAP of five groups under the voltage stimulation of $10 \mathrm{~V}$ was significantly different $(P>0.05)$. ICP/MAP in ADSC group, LI-ESWT group and combined treatment group was significantly higher than that of ED model group; ICP/MAP in combined treatment group was higher than ADSC group and LI-ESWT group. Interaction between LI-ESWT and ADSCs

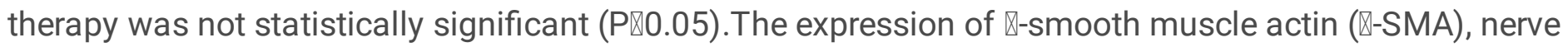
nitricoxide synthase (nNOS) and von Willebrand factor (vWF) in ADSCs treatment group, LI-ESWT group and combined treatment group was higher than those in ED model group $(P<0.05)$.

Conclusions $₫$ Both $A D S C s$ and LI-ESWT therapy can improve the erectile function of rats. The combination of these two methods present better effect on ED than used individually. There is no positive interaction between ADSCs therapy and LI-ESWT. Both ADSCs and LI-ESWT can enhance the expression of a-SMA, nNOS and VWF in corpus cavernosum of ED rats.

\section{Background}

Erectile dysfunction (ED) is defined as the inability of a male to attain and maintain erection of the penis to permit satisfactory sexual intercourse [1], It is one of the most common symptoms affecting the life quality of middle-aged men [2]. ED is more prevalent in men with diabetes mellitus (DM). A Chinese study showed that the prevalence of ED in diabetics in 2010 was about 75.2\% [3]. Diabetes induced erectile dysfunction (DMED), pathogenic manifestations involving endothelial injury, neuropathy, microvascular and fibrousmuscular alterations is usually more serious and difficult to treat than nondiabetic[4, 5]. Phosphodiesterase 5 inhibitors (PDE5Is) was considered to be the first-line therapeutic drug for ED $₫$ however $\square$ PDE5Is showed lower efficacy in treating DMED [6]. Thus, it becomes a key issue of treat DMED to explore the new treatment. 
Adipose-derived stem cells (ADSCs) are stem cells with multiple-differentiation [7] and immunosuppression potential[8] that isolated from adipose tissue. Stem cells have the potential to increase the expression of nerve nitricoxide synthase (nNOS) in cavernous nerves (CN) and are capable of secreting neurotrophic and angiogenic factors [9, 10], Which all make ADSCs posses potential therapeutic effect on ED. However there remains a challenge to fully explore clearly the efficacy of ADSCs therapy.

Low-intensity extracorporeal shockwave therapy (LI-ESWT), is an emerging physical therapy for ED currently due to its ability to improve erectile function by stimulating the corpus cavernosum (CC) in patients with ED. LESW has been demonstrated to enhance gene expression related to angiogenesis and proliferation, and improve tissue neovascularization and regeneration[11]. LI-ESWT has been frequently used in clinical study and has achieved excellent therapeutic effects $[10,12]$.

Because stem cells have the potential to restore the function of nerves $[9,10]$ and endothelium, smooth muscle in corpus cavernosum (CC) [13], and LI-ESWT has the potential to improve penile vascular endothelial function [14], we hypothesized that combining these two treatments would improve recovery effect of erectile function than applying the two treatments separately in a rat DMED model. Therefore, in this study, the purpose of the present study was to investigate the efficacy of ADSCs combined with LI-ESWT therapy for DMED and whether have the significant interaction of these two methods in an streptozotocin (STZ)- induced diabetic rat model of ED.

\section{Methods}

\section{Animals}

A total of 150 male's specific pathogen free (SPF) rats aged 8 weeks old (body weight: 300-370g) were purchased from the Animal Center of Henan province, and this was consistent with animal experimental ethics. 130 rats in the interaction analysis group were fasted for 12 hours, and then intraperitoneally injected with streptozotocin (STZ) (Beijing Dingguo Changsheng Biotechnology Co.Ltd, 55 mg/kg). Blood glucose levels were measured 
at weeks 2, 4, 6 and 8 respectively. Of the 130 rats, 103 rats were diabetic with a randomized blood glucose $\geq 16.7 \mathrm{mmol} / \mathrm{L}$. An intracavernous (IC) injection of phosphate buffer solution (PBS) (Wuhan Seville Biological Technology Co., Ltd) was administered to 20 rats as the normal control group. At 8 weeks after STZ injection apomorphine (APO) test was used to screen the diabetic rats, And the 83 of the 103 (80.58\%) rats with negative APO test results were numbered and randomly assigned to four groups: ED control group (ED) was given IC injection of PBS; ADSCs treatment group (ADSCs) was given IC injection of ADSCs suspension; LI-ESWT treatment group (LI-ESWT) was given cavernous shock wave treatment; ADSCs combined with LI-ESWT treatment group (ADSCs + LI-ESWT) was given cavernous shockwave treatment one day after injection of ADSCs. The control group also received an IC injection of PBS.

The Ethics Review Committee of the Life Science of Zhengzhou University approved the study. Animals were euthanized with carbon dioxide at the conclusion of the experiment. This article does not contain any studies with human participants performed by any of the authors

\section{ADSC isolation and Intracavernous injection}

ADSCs were isolated from the inguinal fats of two 4-week-old SPF SD rats $60080 \mathrm{~g} \square$ and cultured as previous standardized method[15].We use MTT assay to detect the proliferation capacity of ADSCs and found the passage 3 ADSCs proliferate fastest, we also performed osteogenic and adipogenic induction and found passage 3 ADSCs could well differentiate into adipocytes and osteocytes after induction. So we use the passage 3 ADSCs as cell suspensions and then intracavernous injection. Under aseptic conditions, the rats in ADSCs, ED and the normal control group were anesthetized. The penis was exposed in each group, and a 24gauge needle was used to inject a total of $1 \times 106$ ADSCs (dissolved in $100 \mu$ PBS) or only $100 \mu \mathrm{l}$ PBS into the corpus cavernosum (CC). An elastic band was applied to the base of the penis and the pressure was maintained for 2 minutes after the injection.

\section{LI-ESWT treatment}

Rats in the LI-ESWT and ADSCs + LI-ESWT groups were treated with shockwaves. After anesthesia, the penis was drawn out of the prepuce in a supine position. Ultrasonic gel was 
applied on the penis and then the shockwave applicator (HB-ESWT-01 shock wave musculoskeletal pain treatment machine, Seaside Medical Devices Co., Ltd, China) was placed on the penis. A total of 300 (150 on each side of the CC) shocks were delivered at energy level of $0.09 \mathrm{~mJ} / \mathrm{mm}^{2}$ and a frequency of $120 / \mathrm{min}$ during each LI-ESWT session[16]. LI-ESWT was repeated 3 times per week with a day's break in between each session, for a total duration of 3 weeks.

\section{Measurement of erectile function}

Erectile function was assessed 4 weeks after intervention. The carotid artery and cavernous nerve were exposed to measure the mean arterial pressure (MAP) and the intracavernosal pressure (ICP), respectively. The rats were anesthetized in a supine position. The major pelvic ganglion (MPG) and CN were exposed through a midline laparotomy. One of the 21-gauge needles that were connected to PE-tubes with heparinized saline $(250 \mathrm{U} / \mathrm{ml}$, Beijing Solarbio Technology Co., Ltd) was inserted into the carotid artery to measure MAP. The other one was inserted into CC to measure ICP. PE-tubes were connected to the MD3000 signal acquisition (Anhui Zheng Hua biological instrument and Equipment Co., Ltd.) and processing system (Anhui Zheng Hua biological instrument and Equipment Co., Ltd.). The CNs was stimulated using a stainless steel bipolar hook electrode with the following parameters: $10 \mathrm{v}, 20 \mathrm{~Hz}$, with a pulse width of $0.2 \mathrm{~ms}$ for 1 minute. At the same time, the vascular clamp was loosened and the pressure of ICP and MAP were recorded.

\section{Measurement of efficiency and cure rate}

No previous study has demonstrated which level of ICP/MAP is the therapeutic efficiency on ED. Our study used the minimum level of ICP/MAP in the normal group as the standard to calculate the effective rate of ADSCs and LI-ESWT for ED, used the lower limit of 95\% confidence interval (CI) of ICP/MAP in the normal group as the standard to calculate the cure rate of $\mathrm{ADSC}$ and LI-ESWT for $\mathrm{ED}$. The effective rate $=\mathrm{A} / \mathrm{B}(\mathrm{A}=$ the rats number in treatment group when ICP/MAP level were higher than the minimum of ICP/MAP level in the normal 
group and $B=$ the total number of samples in the treatment group). The cure rate $=C / D(C=$ the rats number in treatment group when ICP/MAP level were higher than the 95\%CI lower limit of ICP/MAP level in the normal group and D refers to the total number of samples in the treatment group).

\section{Immunohistochemistry}

For Immunohistochemistry (IH), the penile tissue (midshaft portion) were fixed in fresh 4\% paraformaldehyde (Beijing Solarbio Technology Co., Ltd) for 18 24 hours. The fixed tissues were put in paraffin blocks and cut into $5 \mu \mathrm{m}$ sections before mounting on slides. After dewaxing and antigen repair, the slides were incubated with primary antibodies (Table 1), including mouse anti- $\alpha$ smooth muscle actin ( $\alpha$-SMA, 1:500; Wuhan Seville Biological Technology Co., Ltd), rabbit anti-neuronal nitric oxide synthase (nNOS, 1:200; Wuhan Seville Biological Technology Co., Ltd), rabbit anti-von Willebrand factor (vWF, 1:500; Wuhan Seville Biological Technology Co., Ltd) at $4^{\circ} \mathrm{C}$ overnight, then the sections were rinsed and incubated with secondary antibodies (Table 1). Nuclei were stained by 4', 6-diamidino-2-phenylindole (DAPI, Beyotime Biological Technology Co.,Ltd) for $5 \mathrm{~min}$. Slides were visualized under a fluorescence microscope at 200 and 400 times of visual field. The photographs were then analyzed by Pro Plus6.0 software. Semi quantitatively the protein expression was calculated as the ratio of the fluorescent expression area to the measured area. In addition to, we labeled ADSCs with 5-Bromo-2'-deoxyuridine (Wuhan Seville Biological Technology Co., Ltd) before the ADSCs transplantation, and identified the cells by immunohistochemistry at the end of the experiment to detect the changes of cavernous microstructures in rats.

Table 1 Name, dilutions and source of the primary and secondary antibodies were used in this study

\begin{tabular}{|c|c|c|c|c|c|}
\hline & Primary & Dilution & Secondary & Dilution & Source \\
\hline 1 & nNOS & $1: 200$ & $\begin{array}{l}\text { CY3 Goat anti- } \\
\text { rabbit }\end{array}$ & $1: 300$ & $\begin{array}{c}\text { Wuhan Seville Biological Technology } \\
\text { Co.. Ltd }\end{array}$ \\
\hline 2 & vWF & $1: 500$ & $\begin{array}{l}\text { CY3 Goat anti- } \\
\text { rabbit }\end{array}$ & $1: 300$ & $\begin{array}{c}\text { Wuhan Seville Biological Technology } \\
\text { Co., Ltd }\end{array}$ \\
\hline 3 & $\alpha$-SMA & $1: 500$ & $\begin{array}{l}\text { CY3 Goat anti- } \\
\text { mouse }\end{array}$ & $1: 300$ & $\begin{array}{c}\text { Wuhan Seville Biological Technology } \\
\text { Co., Ltd }\end{array}$ \\
\hline
\end{tabular}


$\alpha$-SMA, $\alpha$-smooth muscle actin; nNOS, nerve nitricoxide synthase; vWF, von Willebrand factor

\section{Statistical analysis}

Statistical analyses were performed with the SPSS 21.0; Measurement data were expressed as mean \pm standard deviation (SD). The comparisons of blood glucose and weight were analyzed using one-way ANOVA or LSD; One-way ANOVA was used to test the difference of ICP/MAP between different groups; Univariate ANOVA was used to test the interaction between ADSC and LI-ESWT; We also performed the additive model to analysis interaction of efficacy by calculating the attributable proportion due to interaction (AP), Additionally, relative excess risk due to interaction (RERI) and the synergy index (SI) were also performed to evaluate interactions. All statistical tests were two-tailed and results were considered significant when $\mathrm{p}<0.05$.

\section{Results}

\section{Characterization and Construction of animal model}

One rat in the ED, one rat in the ADSCs and two rats in the LI-ESWT were dead before the end of experiment (Figure 1). The final blood glucose levels of STZ-induced diabetic rats were remarkably higher compared to PBS-treated control rats $(F=220.51 \square P<0.05)$. The final weights of diabetic rats were markedly lower than normal rats $(F=45.17, P<0.05)$. Among ED, ADSCs, LI-ESWT and ADSCs + LI-ESWT groups, blood glucose levels and body weights were not statistically different (Table 2).

Fig. 1 The construction and intervention of experimental animal models. The number of rats finally enrolled in our statistical analysis in each group were as following: 19 in the normal 
group, 19 in the ED model group, 20 in the ADSCs treatment group, 19 in the LI-ESWT treatment group and 21 in the ADSCs combined with LI-ESWT treatment group

Table 2 Characterization of the animals before and after intervention

\begin{tabular}{cccccc}
\hline Variable & Control & ED & ADSCs & LI-ESWT & $\begin{array}{c}\text { ADSCS+LI- } \\
\text { ESWT }\end{array}$ \\
& $\mathrm{N}=20$ & $\mathrm{~N}=20$ & $\mathrm{~N}=21$ & $\mathrm{~N}=21$ & $\mathrm{~N}=21$ \\
\hline $\begin{array}{c}\text { Initial } \\
\text { weight } \\
\text { (g) }\end{array}$ & $430.19 \pm 32.81$ & $302.9 \pm 33.84$ & $312.53 \pm 44.34$ & $306.05 \pm 32.67$ & $317.6 \pm 35.46$ \\
$\begin{array}{c}\text { Final } \\
\text { weight } \\
\text { (g) }\end{array}$ & $486.75 \pm 46.08$ & $275.14 \pm 35.04^{*}$ & $297.58 \pm 50.51^{*}$ & $278.23 \pm 27.66^{*}$ & $319.36 \pm 42.74^{*}$ \\
$\begin{array}{c}\text { Initial } \\
\text { glucose } \\
\begin{array}{c}\text { mmol/L) } \\
\text { Final }\end{array}\end{array}$ & $6.4 \pm 1.09$ & $30.35 \pm 3.18$ & $29.28 \pm 4.24$ & $30.32 \pm 2.6$ & $28.85 \pm 3.88$ \\
$\begin{array}{c}\text { glucose } \\
\text { mmol/L) }\end{array}$ & $6.70 \pm 1.33$ & $28.19 \pm 4.12^{*}$ & $29.58 \pm 4.2^{*}$ & $29.86 \pm 3.53^{*}$ & $28.68 \pm 5.35^{*}$ \\
\hline
\end{tabular}

Values are expressed as mean \pm SD

ED, erectile dysfunction;ADSCs, Adipose-derived stem cells; LI-ESWT, low-intensity extracorporeal shockwave therapy

* $P<0.05$ when compared with control group

\section{The Characterization of ADSCs}

The proliferation capacity of different passage ADSCs were found the passage 3 ADSCs proliferate fastest (Figure 2a). Compared with the normal ADSCs (A;C), passage 3 ADSCs could well differentiate into adipocytes(B) and osteocytes(D) after induction. Osteogenic and adipogenic differentiations demonstrated that ADSCs have the potential for multipotency (Figure 2b).

Fig. 2 The characterization of the ADSCs. (a) The proliferation capacity of different passage ADSCs. (b) Photomicrographs showing osteogenic (Alizarin Red staining) and adipogenic (Oil Red O staining) differentiations of ADSCs. A, Before adipogenic induction; B, After adipogenic induction; C, before osteogenic induction; D,After osteogenic induction 


\section{Effects of ADSCs and LI-ESWT on erectile function in rats}

The typical images of ICP/MAP in each group were shown in Figure 3a. The ICP-to-MAP ratios were showed significant decreases in ED group compared to other groups $(P<0.05)$ (Figure 3b). ADSCs, LI-ESWT and ADSCs + LI-ESWT treatment partially ameliorated erectile dysfunction compared with DM group $(P<0.05)$ (Figure 3b). ADSCs + LI-ESWT treatment, however, displayed significant improvement in erectile function compared with ADSC or LIESWT mono-therapy group $(P<0.05)$ (Figure $3 \mathrm{~b})$. The number (rate) of effective and cure in ADSCs, LI-ESWT and ADSCs + LI-ESWT treatment groups was 7(35.0\%), 3(15.8\%), $12(57.1 \%)$ and $0(0 \%), 1(5.3 \%), 4(19.0 \%)$ respectively (Figure 3c). This means all treatment methods are effective, but still a larger proportion of ICP/MAP could not reduce to a standard level and make function of ED complete recovery.

Fig. 3 The erectile function of each group. (a) Representative images of intracavernous pressure (ICP) and mean arterial pressure (MAP) in control (A), ED (B), ADSCs (C), LI-ESWT (D), ADSCs +LI-ESWT (E) groups respectively; (b) Results of ratio ICP to mean MAP of each group. ${ }^{*} P<0.05$ when compared with ED group. $\& P<0.05$ when compared with ADSCs +LIESWT group; (c) The number (rate) of effective and cure in each group

\section{Interaction analysis}

Interaction analysis showed that the single effect of ADSCs and LI-ESWT on ICP/MAP was significant $(P<0.001)$, but no interaction $(F=1.587, P=0.212)$ between these two methods (Table 3). The value of RERI, AP, SI are 11.936, 0.497 and 2.079 respectively, implies that there was no additive interaction of efficiency between ADSCs and LI-ESWT treatment (Table $3)$. 


\begin{tabular}{ccccc}
\hline Model & \multicolumn{4}{c}{ Measurement } \\
\hline Univariate ANOVA & Variable & Mean square & F & $P$ \\
\cline { 2 - 5 } & ADSCs & 0.371 & 28.406 & $0.000^{*}$ \\
& LI-ESWT & 0.282 & 21.601 & $0.000^{*}$ \\
& ADSCs+ LI-ESWT & 0.021 & 1.587 & 0.212 \\
\hline Additive model & Variable & Estimate & Lower (95\%CI) & Upper (95\%CI) \\
\cline { 2 - 5 } & RERI & 11.936 & -19.855 & 43.727 \\
& AP & 0.497 & -0.105 & 1.099 \\
& SI & 2.079 & 0.57 & 7.583 \\
\hline
\end{tabular}

ADSC, Adipose-derived stem cells; LI-ESWT, low-intensity extracorporeal shockwave therapy; RERI, relative excess risk due to interaction; AP, the attributable proportion due to interaction; $\mathrm{S}$, the synergy index

* $P<0.001$ was considered statistically significant

\section{Expression of $\alpha-S M A, n N O S$ and vWF in Corpus Cavernosum}

\section{$\alpha-S M A$ expression in the Corpus Cavernosum}

The expression of $\alpha$-SMA in control, ED, ADSCs, LI-ESWT and ADSCs + LI-ESWT were $(0.06 \pm 0.02),(0.01 \pm 0.01),(0.05 \pm 0.01),(0.05 \pm 0.01)$ and $(0.06 \pm 0.01)$, respectively (Figure $4 \mathrm{a})$. The comparison between each two groups among five groups showed that the expression of $\alpha$ SMA in the other 4 groups were higher than that of ED model group $(P<0.05)$ (Figure $4 \mathrm{~b})$; The expression of $\alpha$-SMA in ADSCs was lower than LI-ESWT and ADSCs+LI-ESWT $(P<0.05)$ (Figure 4b). 
Fig. 4 The nNOS expression in Corpus Cavernosum (CC). (a) Representative images of nNOS expression in control group, ED, ADSCs, LI-ESWT, ADSCs +LI-ESWT respectively, original magnification: $\times 200$; (b) Expression level of $\alpha$-SMA in each group, in each column and expressed as mean \pm standard deviation, $* P<0.05$ when compared with ED group. $\& P<0.05$ when compared with ADSCs +LI-ESWT group

\section{vWF expression in the Corpus Cavernosum}

The expression of vWF in control, ED, ADSCs, LI-ESWT and ADSCs+LI-ESWT were $(0.25 \pm 0.03),(0.04 \pm 0.06),(0.18 \pm 0.11),(0.2 \pm 0.08)$ and $(0.13 \pm 0.07)$, respectively (Figure 5a). The comparison between each two groups among five groups showed that the expression of vWF in the other 4 groups were higher than that of ED model group $(P<0.05)$ (Figure 5b); The expression of vWF in ADSCs, LI-ESWT and ADSCs+LI-ESWT has no difference $(P>0.05)$ (Figure 5b).

Fig. 5 The vWF expression in Corpus Cavernosum (CC). (a) Representative images of vWF expression in control group, ED, ADSCs, LI-ESWT, ADSCs +LI-ESWT respectively, original magnification: $\times 200$; (b) Expression level of vWF in each group, in each column and expressed as mean \pm standard deviation, $* P<0.05$ when compared with ED group. $\& P<0.05$ when compared with ADSCs +LI-ESWT group

\section{nNOS expression in the Corpus Cavernosum}

The expression of nNOS in control, ED, ADSCs, LI-ESWT and ADSCs+LI-ESWT were $(0.33 \pm 0.08),(0.08 \pm 0.07),(0.25 \pm 0.08),(0.19 \pm 0.06)$ and $(0.29 \pm 0.04)$, respectively (Figure 6a). The comparison between each two groups among five groups showed that the expression of nNOS in the other 4 groups were higher than that of ED model group $(P<0.05)$ (Figure $6 \mathrm{~b})$; The expression of nNOS in ADSCs, LI-ESWT and ADSCs+LI-ESWT has no difference $(P>0.05)$ (Figure 6b). 
Fig. 6 The nNOS expression in Corpus Cavernosum (CC). (a) Representative images of nNOS expression in control group, ED, ADSCs, LI-ESWT, ADSCs +LI-ESWT respectively, original magnification: $\times 200$; (b) Expression level of nNOS in each group, in each column and expressed as mean \pm standard deviation, $* P<0.05$ when compared with ED group. $\& P<0.05$ when compared with ADSCs +LI-ESWT group

\section{Discussion}

In this study, we confirmed that both ADSC and LI-ESWT treatment significantly ameliorated ED in STZ-induced diabetic rats, and ADSCs combined with LI-ESWT treatment showed better effects which similar with to normal rats. Our results also demonstrated that both ADSCs and LI-ESWT could enhance the expression of [-SMA, nNOS and vWF in CC of ED rats.

ED seriously affects the patient's mental health and the quality of life of patients and their partners. Although clinically treatment, such as PDE5Is, have been used to treat ED, however! PDE5Is showed less efficacy in treating DMED[17] and the treatment of stem cell therapy and LI-ESWT therapy has been two emerging therapies for ED currently.

Stem cells play an important role in the treatment of ED due to their multipledifferentiation and immunosuppression potential, and have already been explored in different ED models[13, 18, 19]. As for ED therapy, some study has suggested that ADSCs could improve the therapeutic effect of erectile function in rat models of DMED[20]. In the present study, expression increased of प-SMA, vWF and nNOS in CC were observed in ADSCs-injected diabetic rats. These results are consistent with previous findings about diabetic rats [21].

In this study, we explored another treatment to treat the DMED, namely LI-ESWT. Qiu et al utilized LI-ESWT and found that the treatment was able to restore the function of the erectile tissues. However, while our study also produced consistent results that LI-ESWT could improve the therapeutic effect of erectile function in rat models of DMED. LI-ESWT has been known could significantly improve penile vascular endothelial function and penile flow velocity $[14,22,23]$, in addition, DMED involves dysfunction of the nerves, endothelium, and smooth 
muscle, which may partially explained why the LI-ESWT could improve the therapeutic effect of erectile function in our study.

Although, both ADSCs and LI-ESWT therapies could improve the erectile function, the combined effect of ADSCs and LI-ESWT for DMED are yet to be determined. Thus, our study hypothesized that combining the use of ADSCs and LI-ESWT would significantly improve the treatment of DMED. Our results suggest that combining ADSCs and LI-ESWT could play an important role in the treatment of the DMED. We also explored the interaction between ADSCs and LI-ESWT from a statistical point of view. However, the exact mechanism of erectile function recovery due to dual therapeutics utilizing ADSCs and LI-ESWT still remains unclear.

As we all know, erectile function was closely related to endothelium, smooth muscle and nerves of CC[24], so we selected three relevant markers (口-SMA, vWF and nNOS). Because the key of our research is whether have the combined effcet and interaction between ADSCs and LI-ESWT and we only need qualitative observation whether the different treatment methods could promote the expression of markers, so we only used the IH and it's semiquantitative ability without western blot. The results of our study showed that ADSCs, LI-ESWT and their combination could increase the expression of प-SMA, vWF and nNOS, suggest that the combination of ADSCs and LI-ESWT could play a key role in improve the pathological status of endothelium, smooth muscle and nerves in corpus cavernosum. The precise mechanisms whereby ADSCs and LI-ESWT on increasing the expression of प-SMA, vWF and nNOS in the penile nerve are yet to be determined, but it is speculated that ADSCs and LI-ESWT might contribute to the recovery of the cavernous nerve or the growth of endothelial cells $[25,26]$. While the precise molecular mechanism underlying the increased efficacy of the dual treatment is not entirely clear, our results clearly demonstrate that a combination therapy of injecting ADSCs and applying LI-ESWT was more effective than separate treatments at enhancing erectile function.

Although our study demonstrated improved erectile function with a combination therapy of ADSCs and LI-ESWT, investigations should be carried out in more ED animal models and to better evaluate the therapeutic effects and safety for potential clinical application. Our study 
used 4 weeks after initial ADSCs and LI-ESWT as the end time point, so it is yet unknown if the reported effects last as long as 6 months or 1 year. Moreover, a limitation of our work is that we use 5-Bromo-2'-deoxyuridine to mark ADSCs, but no labeled cells were detected. The most probable cause is that ADSCs will migrate and play a role in other tissues[27], another cause is that the longevity and specificity of 5-Bromo-2'-deoxyuridine decreases substantially over time[28]. Therefore, the transformation and outcome of the transplanted ADSCs need to be further investigate, more efficient and reliable stem cell labeling techniques are also needed.

\section{Conclusion}

We found that ADSCs and LI-ESWT can improve the erectile function of rats, the combination therapy present better effect on ameliorates ED and pathological changes than single therapy in DMED. Even if only the short-term therapeutic effect were observed, suggesting ADSCs combined with LI-ESWT will be a more promising way for DMED treatment and opening a new horizon to the development of clinical applications. Furthermore, ADSCs and LI-ESWT can improve the penile pathological status by enhance the expression of $\square-S M A$, nNOS and vWF in CC of ED rats. More mechanistic investigations should be carried out and better to evaluate the cellular and molecular mechanisms of action, it will help to find further applications of this treatment in potential clinical application.

\section{Abbreviations}

DM: Diabetes mellitus

ED: erectile dysfunction

ADSCs: Adipose-derived stem cells

LI-ESWT: low-intensity extracorporeal shockwave therapy

DMED: diabetes induced erectile dysfunction 
ICP: intracavernous pressure

MAP: mean arterial pressure

IH: immunohistochemistry

[-SMA: - -smooth muscle actin

nNOS: nerve nitricoxide synthase

vWF: von Willebrand factor

PDE5Is: Phosphodiesterase 5 inhibitors

STZ: streptozotocin

PBS: phosphate buffer solution

APO: apomorphine

\section{Declarations}

\section{Ethics approval and consent to participate}

The Ethics Review Committee of the Life Science of Zhengzhou University approved the study. All procedures performed in studies involving animals were in accordance with the ethical standards of the institution or practice at which the studies were conducted.

\section{Consent for publication}

Not applicable.

\section{Availability of data and material}

Data sharing is not applicable to this article as no datasets were generated or analysed during the current study.

\section{Competing interests}

The authors declare that they have no competing interests. 


\section{Funding}

This study was funded by the docking project of postdoctoral research and development base in Henan Province. These foundations did not interfere in the design of the study and collection, analysis, and interpretation of data and in writing the manuscript.

\section{Authors' contributions}

W-D Z conceived the study. D-K L did the experiments. D-K L and Y-C G researched data, Q-L H, M-Y G, W-H, Q-L Z and C-D Z contributed to discussion and H-Z T reviewed the manuscript. OG contributed to discussion. D-K L wrote the manuscript.

\section{Acknowledgements}

Not applicable.

\section{Authors' Information}

\section{Affiliations}

Department of Epidemiology, School of Public Health, Zhengzhou University, Zhengzhou, Henan, 450001,China

Department of Urology, Zhengzhou Third People's Hospital, Zhengzhou, Henan, 450000,China

Department of internal medicine, Beaumont Health System, Royal Oak, MI

\section{Contributions}

All authors contributed to the design and writing of the paper. All authors have read and approved the final manuscript.

\section{Corresponding author}

Correspondence to Zhengzhou University

\section{References}

1. NIH Consensus Conference. Impotence. NIH Consensus Development Panel on Impotence. Jama. 1993;270(1):83-90. 
2. Montorsi F, Adaikan G, Becher E, Giuliano F, Khoury S, Lue TF, et al. Summary of the recommendations on sexual dysfunctions in men. J Sex Med. 2010;7(11):3572-88.

3. Yang G, Pan C, Lu J. Prevalence of erectile dysfunction among Chinese men with type 2 diabetes mellitus. Int J Impot Res. 2010;22(5):310-7.

4. Hatzimouratidis K, Hatzichristou D. How to treat erectile dysfunction in men with diabetes: from pathophysiology to treatment. Curr Diab Rep. 2014;14(11):545.

5. Thorve VS, Kshirsagar AD, Vyawahare NS, Joshi VS, Ingale KG, Mohite RJ. Diabetes-induced erectile dysfunction: epidemiology, pathophysiology and management. J Diabetes Complications. 2011;25(2):129-36.

6. Goldstein I, Lue TF, Padma-Nathan H, Rosen RC, Steers WD, Wicker PA, et al. Oral sildenafil in the treatment of erectile dysfunction. 1998. The Journal of urology. 2002;167(2 Pt 2):1197-203; discussion 204.

7. Suga H, Glotzbach JP, Sorkin M, Longaker MT, Gurtner GC. Paracrine mechanism of angiogenesis in adipose-derived stem cell transplantation. Ann Plast Surg. 2014;72(2):234-41.

8. Lin CS, Lin G, Lue TF. Allogeneic and xenogeneic transplantation of adipose-derived stem cells in immunocompetent recipients without immunosuppressants. Stem Cells Dev. 2012;21(15):2770-8.

9. Piao S, Kim IG, Lee JY, Hong SH, Kim SW, Hwang TK, et al. Therapeutic effect of adipose-derived stem cells and BDNF-immobilized PLGA membrane in a rat model of cavernous nerve injury. J Sex Med. 2012;9(8):1968-79.

10. Kim IG, Piao S, Lee JY, Hong SH, Hwang TK, Kim SW, et al. Effect of an adipose-derived stem cell and nerve growth factor-incorporated hydrogel on recovery of erectile function in a rat model of cavernous nerve injury. Tissue Eng Part A. 2013;19(1-2):14-23.

11. Wang HJ, Cheng JH, Chuang YC. Potential applications of low-energy shock waves in functional urology. Int J Urol. 2017;24(8):573-81.

12. Lu Z, Lin G, Reed-Maldonado A, Wang C, Lee YC, Lue TF. Low-intensity Extracorporeal Shock Wave Treatment Improves Erectile Function: A Systematic Review and Meta-analysis. Eur Urol. 2017;71(2):223-33.

13. Albersen M, Fandel TM, Lin G, Wang G, Banie L, Lin CS, et al. Injections of adipose tissue-derived stem cells and stem cell lysate improve recovery of erectile function in a rat model of cavernous nerve injury. J Sex Med. 2010;7(10):3331-40.

14. Mittermayr R, Hartinger J, Antonic V, Meinl A, Pfeifer S, Stojadinovic A, et al. Extracorporeal shock wave therapy (ESWT) minimizes ischemic tissue necrosis irrespective of application time and promotes tissue revascularization by stimulating angiogenesis. Ann Surg. 2011;253(5):1024-32.

15. Yu G, Wu X, Kilroy G, Halvorsen YD, Gimble JM, Floyd ZE. Isolation of murine adipose-derived stem cells. Methods Mol Biol. 2011;702:29-36.

16. Vardi Y, Appel B, Jacob G, Massarwi O, Gruenwald I. Can low-intensity extracorporeal shockwave therapy improve erectile function? A 6-month follow-up pilot study in patients with organic erectile dysfunction. Eur Urol. 2010;58(2):243-8. 
17. Kaiho Y, Yamashita S, Arai Y. Optimization of sexual function outcome after radical prostatectomy using phosphodiesterase type 5 inhibitors. Int J Urol. 2013;20(3):285-9.

18. Fall PA, Izikki M, Tu L, Swieb S, Giuliano F, Bernabe J, et al. Apoptosis and effects of intracavernous bone marrow cell injection in a rat model of postprostatectomy erectile dysfunction. Eur Urol. 2009;56(4):716-25.

19. Bae JH, Shrestha KR, Park YH, Kim IG, Piao S, Jung AR, et al. Comparison between subcutaneous injection of basic fibroblast growth factor-hydrogel and intracavernous injection of adipose-derived stem cells in a rat model of cavernous nerve injury. Urology. 2014;84(5):1248 e1-7.

20. Liu G, Sun X, Bian J, Wu R, Guan X, Ouyang B, et al. Correction of diabetic erectile dysfunction with adipose derived stem cells modified with the vascular endothelial growth factor gene in a rodent diabetic model. PLoS One. 2013;8(8):e72790.

21. Zhou F, Hui Y, Xin H, Xu YD, Lei HE, Yang BC, et al. Therapeutic effects of adipose-derived stem cellsbased microtissues on erectile dysfunction in streptozotocin-induced diabetic rats. Asian $\mathrm{J}$ Androl. 2017;19(1):91-7.

22. Nishida T, Shimokawa H, Oi K, Tatewaki H, Uwatoku T, Abe K, et al. Extracorporeal cardiac shock wave therapy markedly ameliorates ischemia-induced myocardial dysfunction in pigs in vivo. Circulation. 2004;110(19):3055-61.

23. Vardi Y, Appel B, Kilchevsky A, Gruenwald I. Does low intensity extracorporeal shock wave therapy have a physiological effect on erectile function? Short-term results of a randomized, double-blind, sham controlled study. J Urol. 2012;187(5):1769-75.

24. Lin CS, Xin ZC, Wang Z, Deng C, Huang YC, Lin G, et al. Stem cell therapy for erectile dysfunction: a critical review. Stem Cells Dev. 2012;21(3):343-51.

25. Kang SK, Lee DH, Bae YC, Kim HK, Baik SY, Jung JS. Improvement of neurological deficits by intracerebral transplantation of human adipose tissue-derived stromal cells after cerebral ischemia in rats. Exp Neurol. 2003;183(2):355-66.

26. Nurzynska D, Di Meglio F, Castaldo C, Arcucci A, Marlinghaus E, Russo S, et al. Shock waves activate in vitro cultured progenitors and precursors of cardiac cell lineages from the human heart. Ultrasound Med Biol. 2008;34(2):334-42.

27. Fandel TM, Albersen M, Lin G, Qiu X, Ning H, Banie L, et al. Recruitment of intracavernously injected adipose-derived stem cells to the major pelvic ganglion improves erectile function in a rat model of cavernous nerve injury. Eur Urol. 2012;61(1):201-10.

28. Li N, Yang H, Lu L, Duan C, Zhao C, Zhao H. Comparison of the labeling efficiency of BrdU, Dil and FISH labeling techniques in bone marrow stromal cells. Brain Res. 2008;1215:11-9.

\section{Tables}

Due to technical limitations, the tables are only available as a download in the supplemental files section 


\section{Figures}

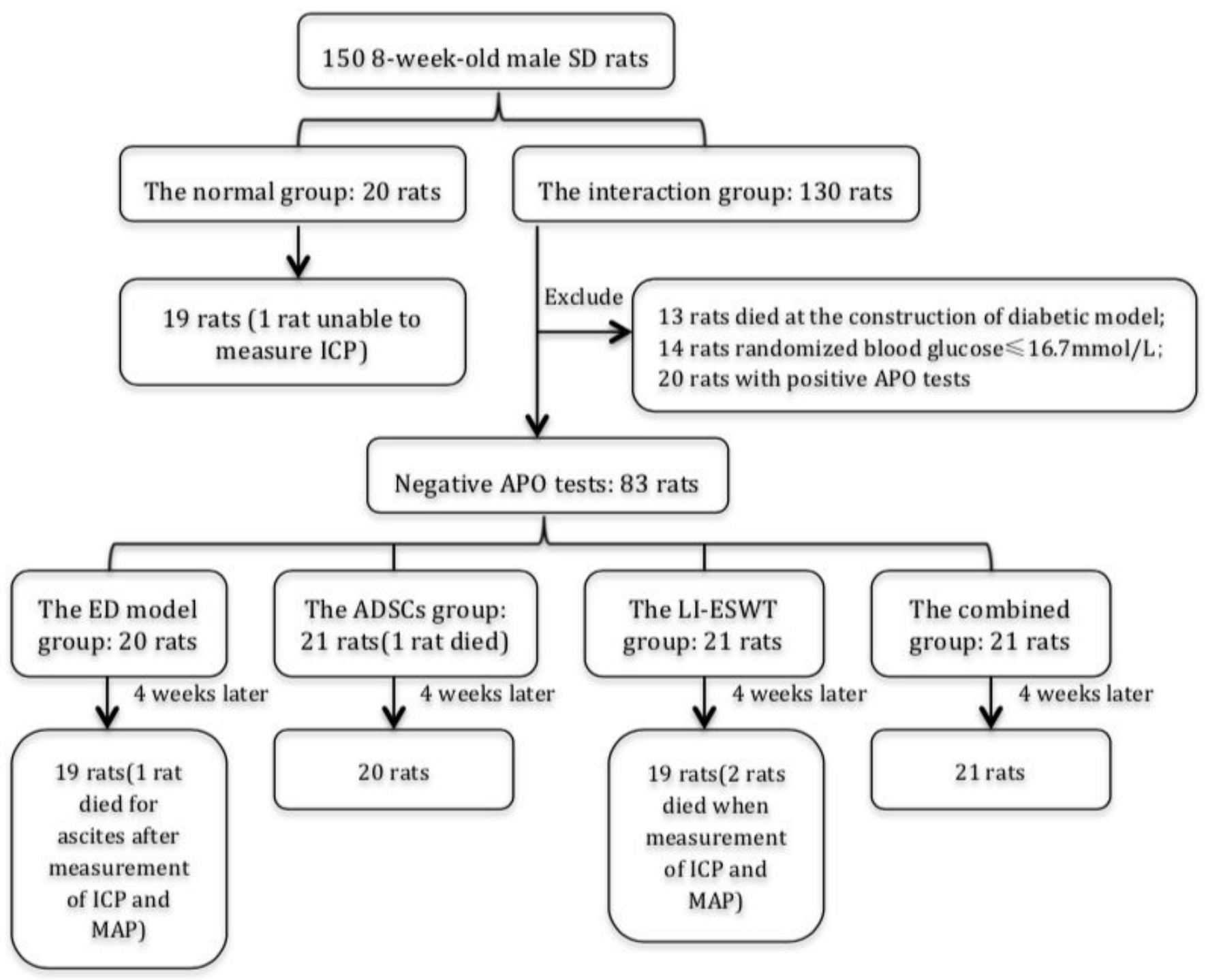

\section{Figure 1}

The construction and intervention of experimental animal models. The number of rats finally enrolled in our statistical analysis in each group were as following: 19 in the normal group, 19 in the ED model group, 20 in the ADSCs treatment group, 19 in the LI-ESWT treatment group and 21 in the ADSCs combined with LI-ESWT treatment group 

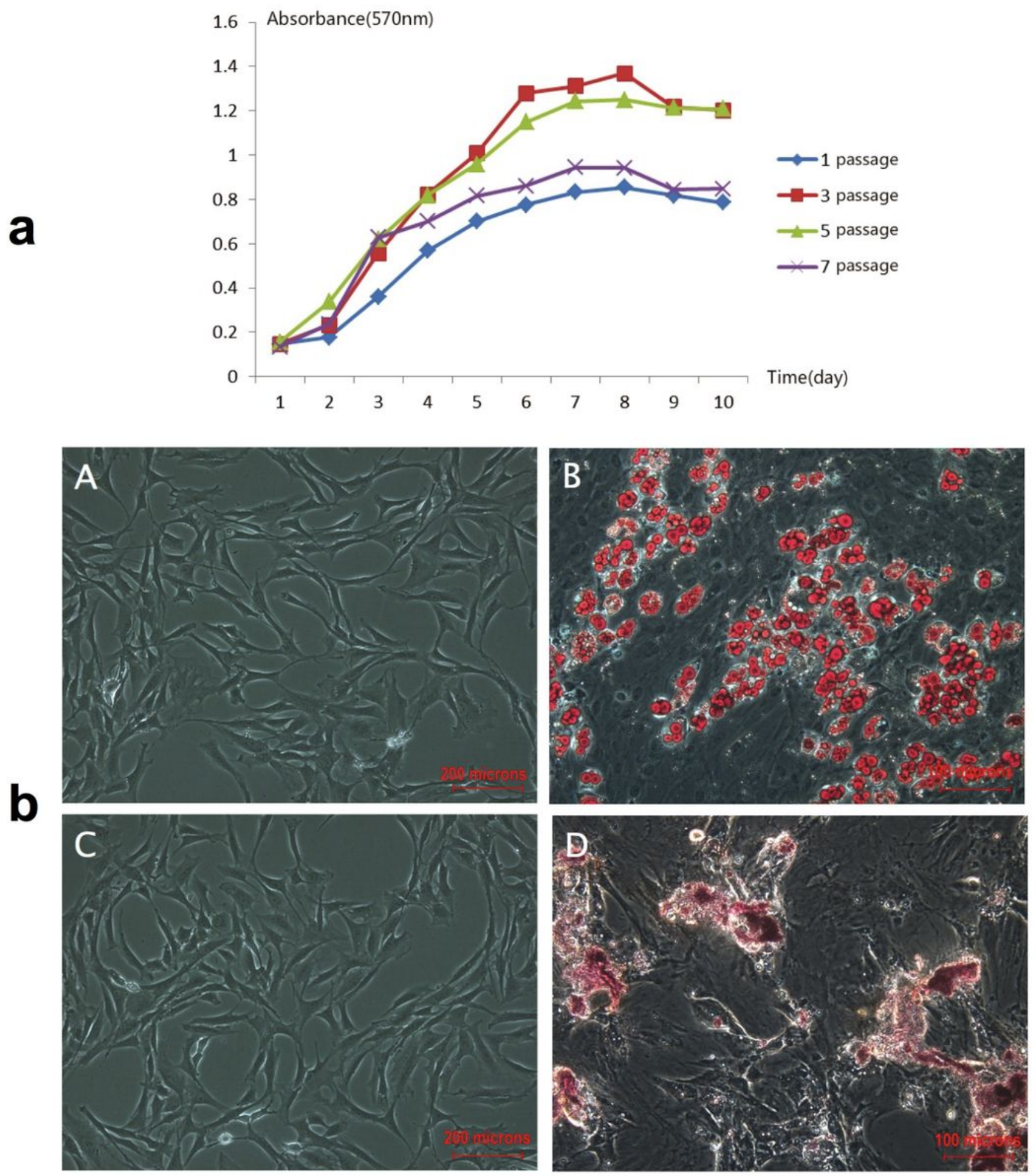

Figure 2

The characterization of the ADSCs. (a) The proliferation capacity of different passage ADSCs. (b) Photomicrographs showing osteogenic (Alizarin Red staining) and adipogenic (Oil Red $O$ staining) differentiations of ADSCs. A, Before adipogenic induction; B, After adipogenic induction; C, before osteogenic induction; D,After osteogenic induction 

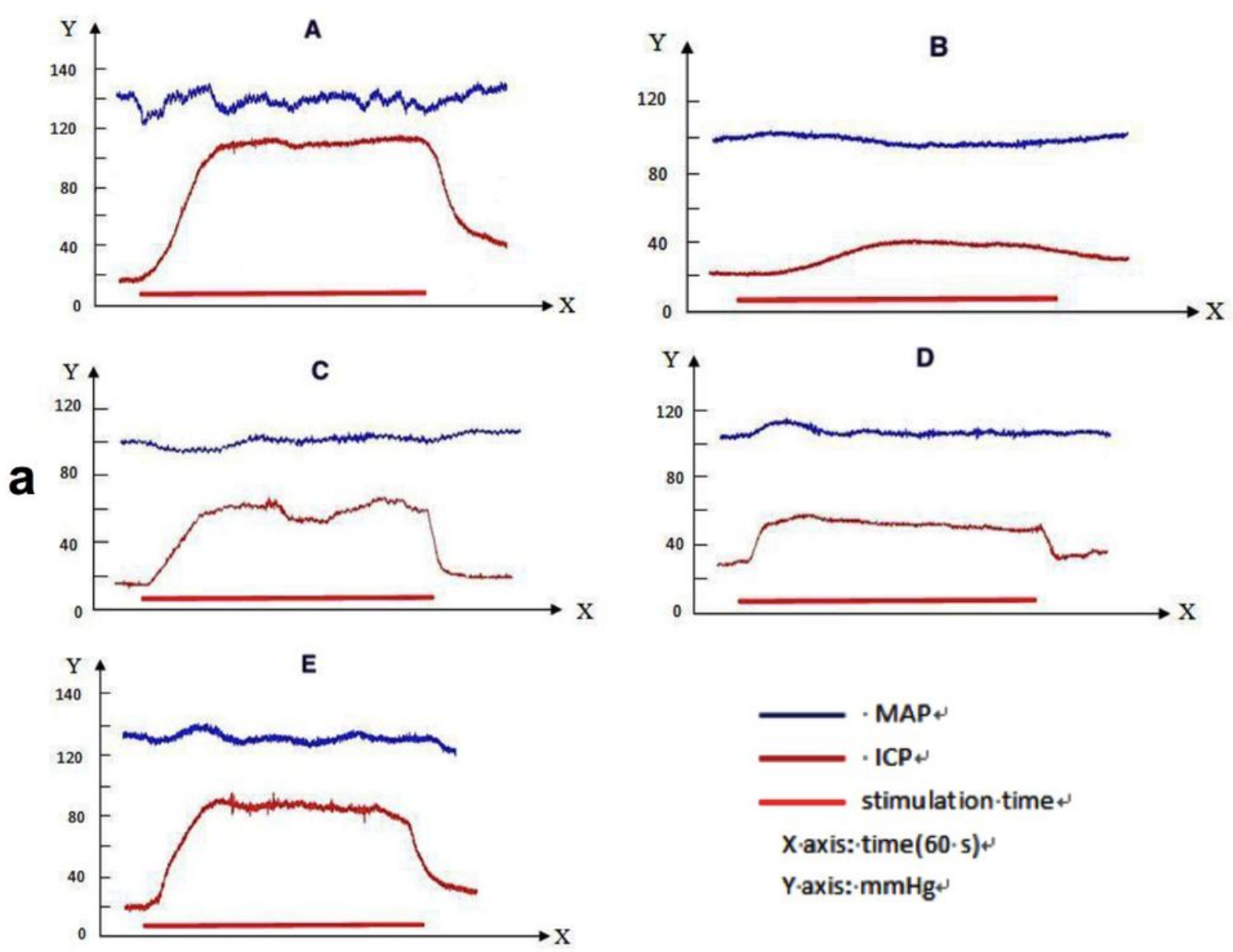

X-axis:-time (60.s)

Y-axis:-mmHg
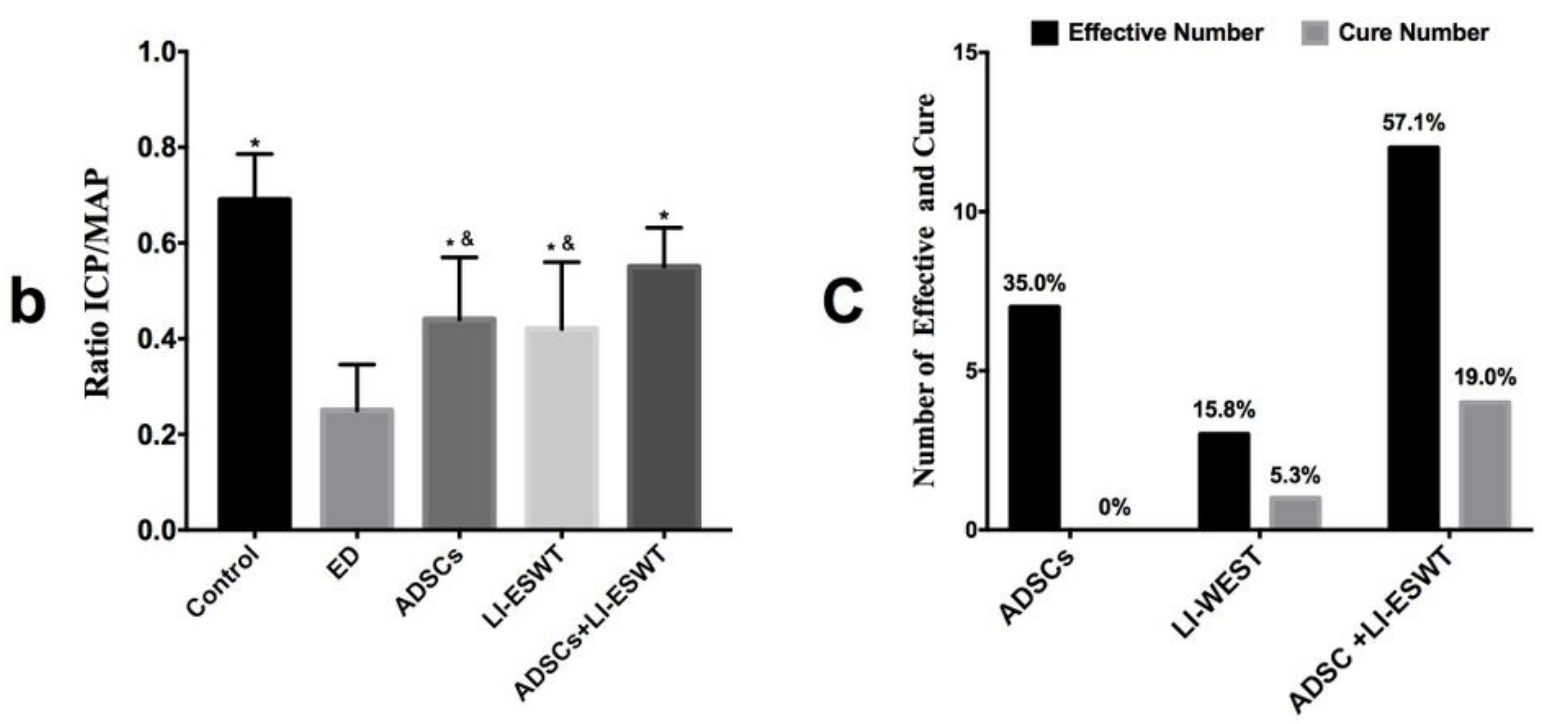

\section{Figure 3}

The erectile function of each group. (a) Representative images of intracavernous pressure (ICP) and mean arterial pressure (MAP) in control (A), ED (B), ADSCs (C), LI-ESWT (D), ADSCs +LI-ESWT (E) groups respectively; (b) Results of ratio ICP to mean MAP of each group. ${ }^{*}<<0.05$ when compared with ED group. $\& \mathrm{P}<0.05$ when compared with ADSCs +LI-ESWT group; (c) The number (rate) of effective and cure in each group 

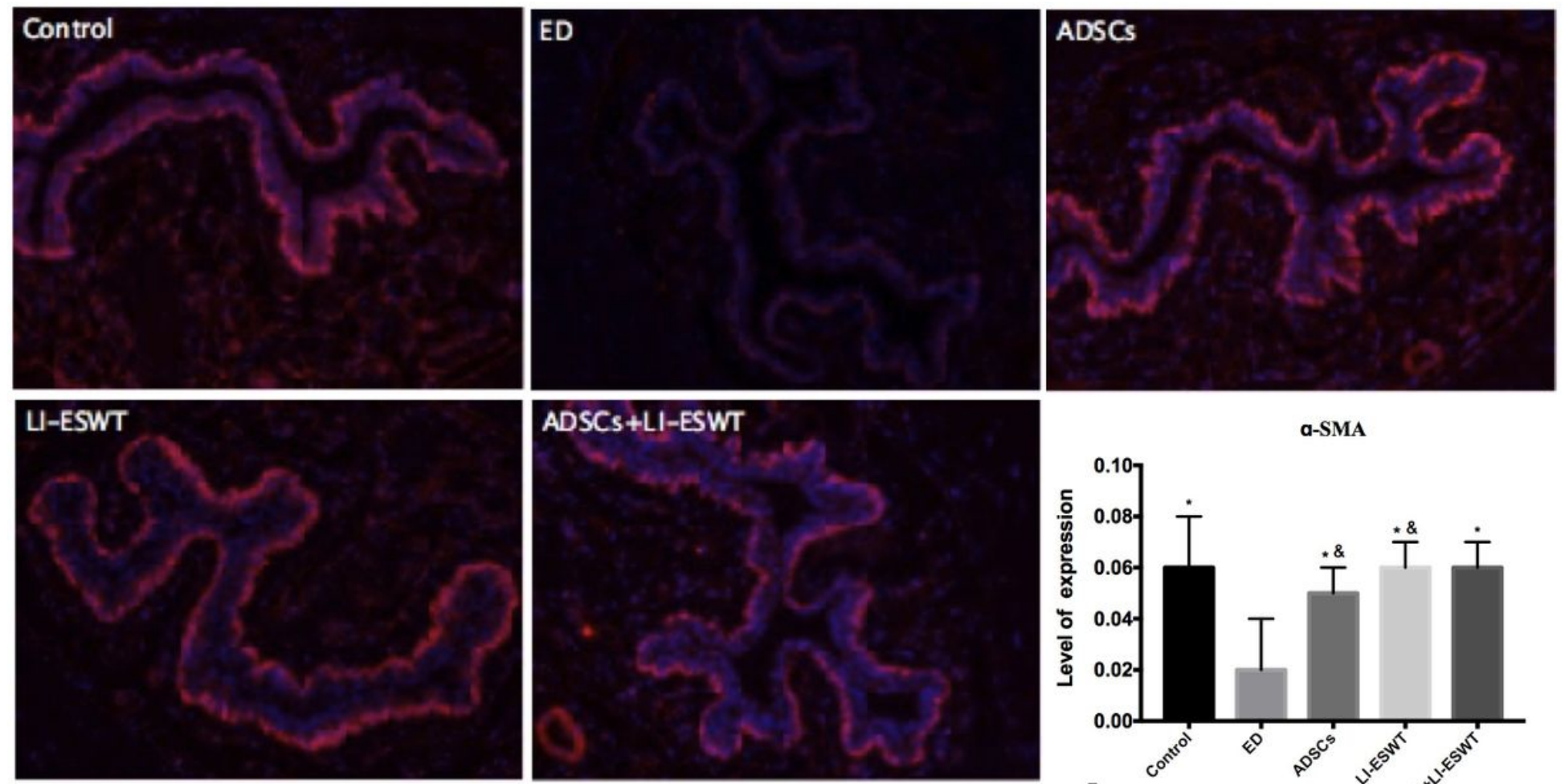

a

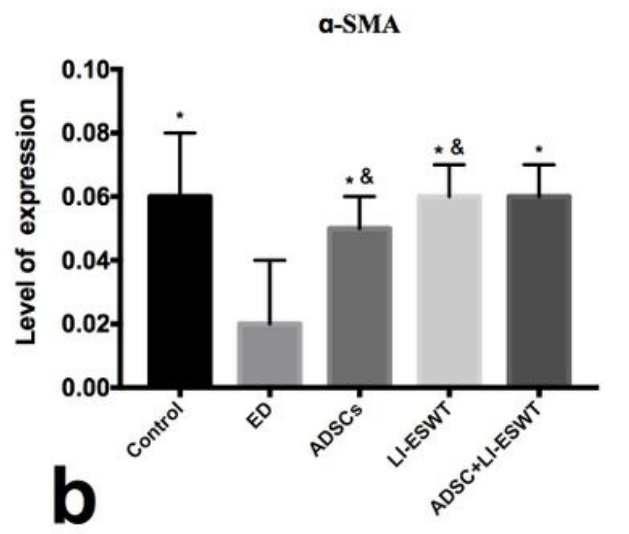

\section{Figure 4}

The nNOS expression in Corpus Cavernosum (CC). (a) Representative images of nNOS expression in control group, ED, ADSCs, LI-ESWT, ADSCs +LI-ESWT respectively, original magnification: ×200; (b) Expression level of a-SMA in each group, in each column and expressed as mean \pm standard deviation, ${ }^{*} \mathrm{P}<0.05$ when compared with ED group. \&P $<0.05$ when compared with ADSCs +LI-ESWT group 

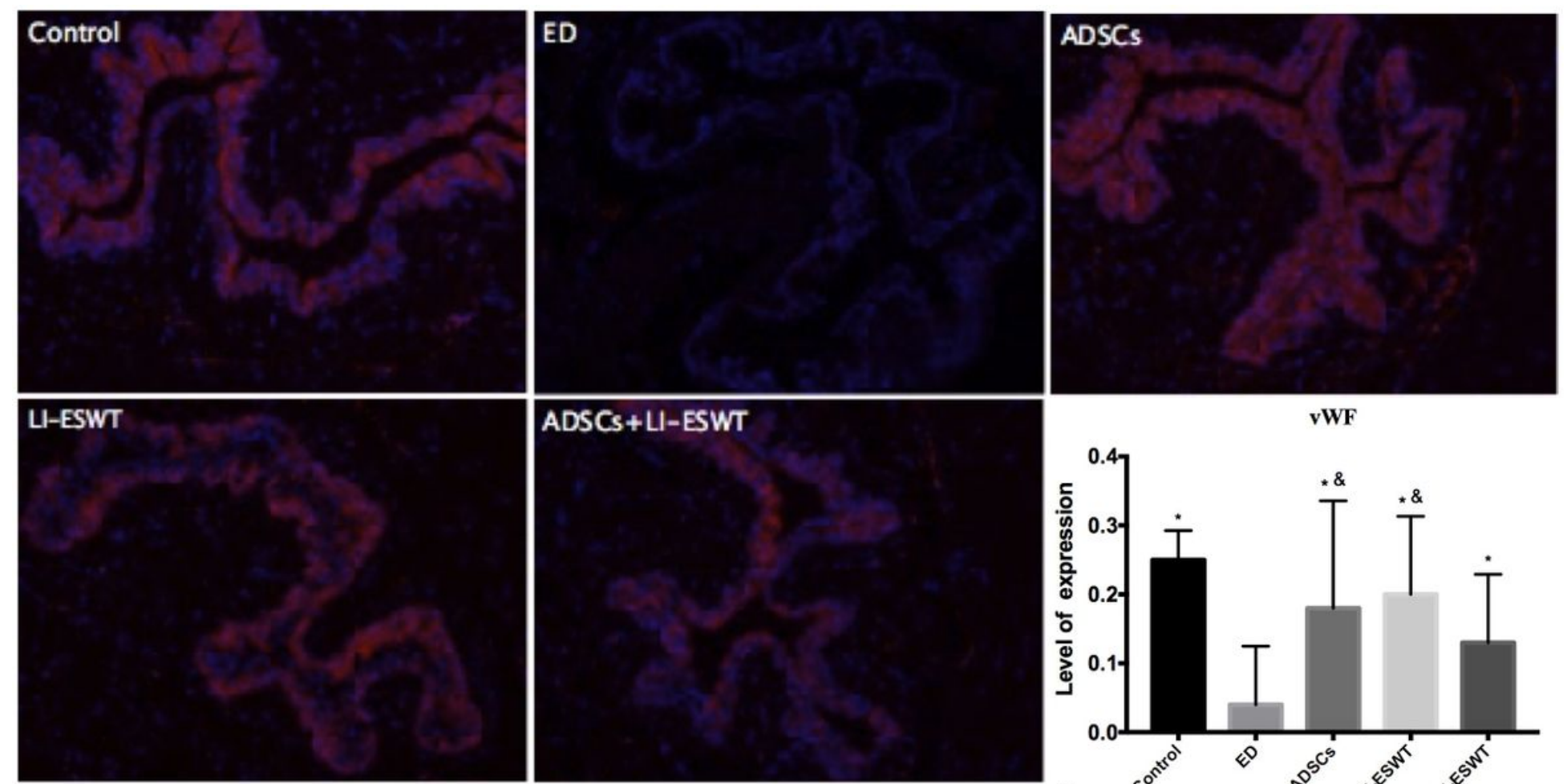

a
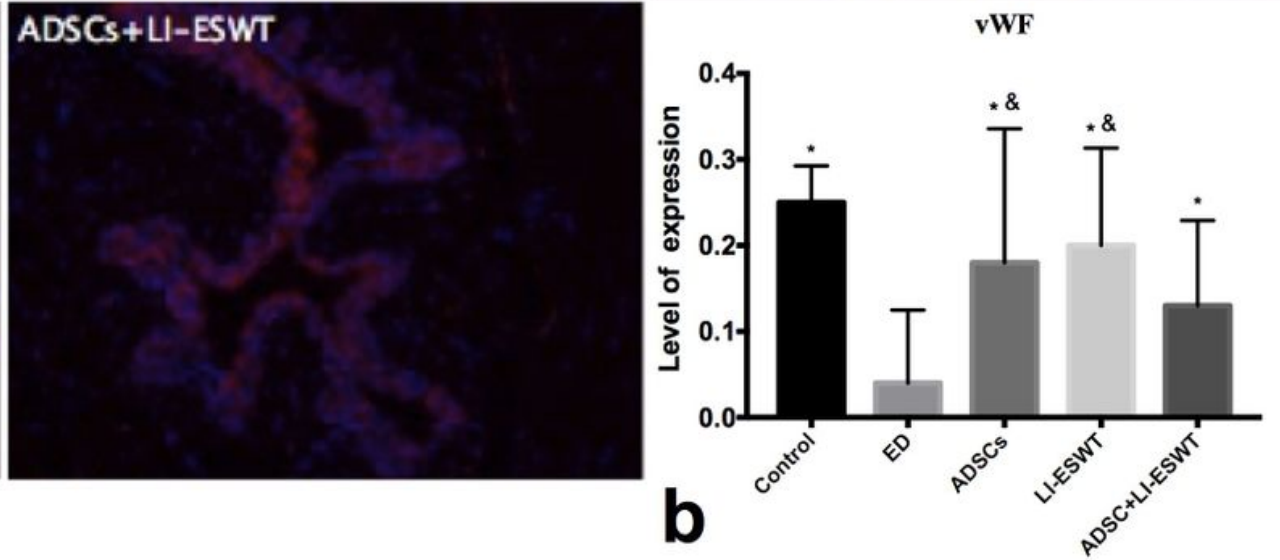

\section{Figure 5}

The vWF expression in Corpus Cavernosum (CC). (a) Representative images of vWF expression in control group, ED, ADSCs, LI-ESWT, ADSCs +LI-ESWT respectively, original magnification: $\times 200$; (b) Expression level of $\mathrm{vWF}$ in each group, in each column and expressed as mean \pm standard deviation, $* \mathrm{P}<0.05$ when compared with ED group. \&P $<0.05$ when compared with ADSCs +LI-ESWT group 

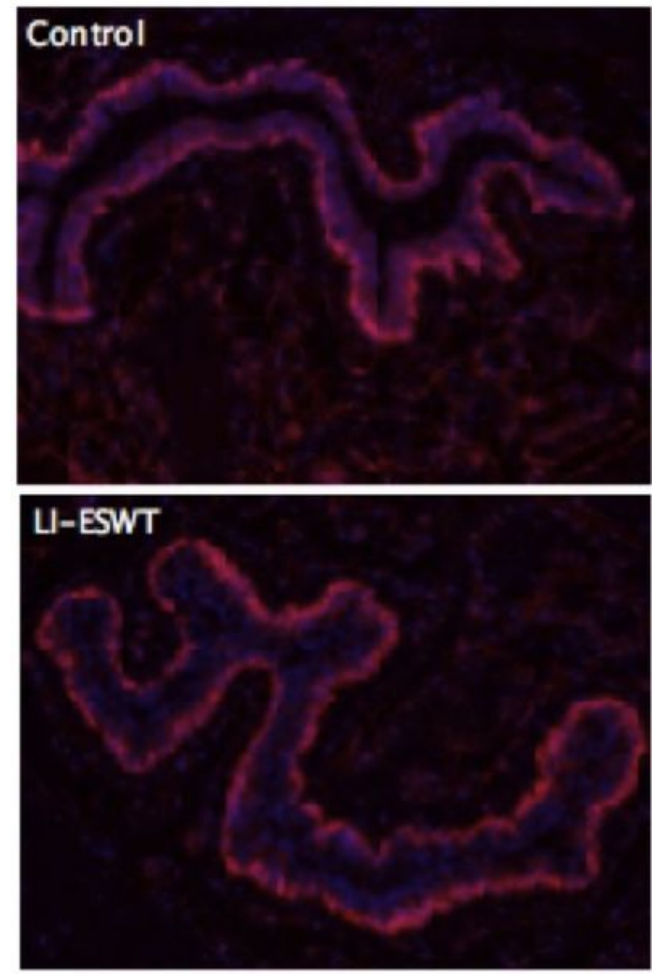

a
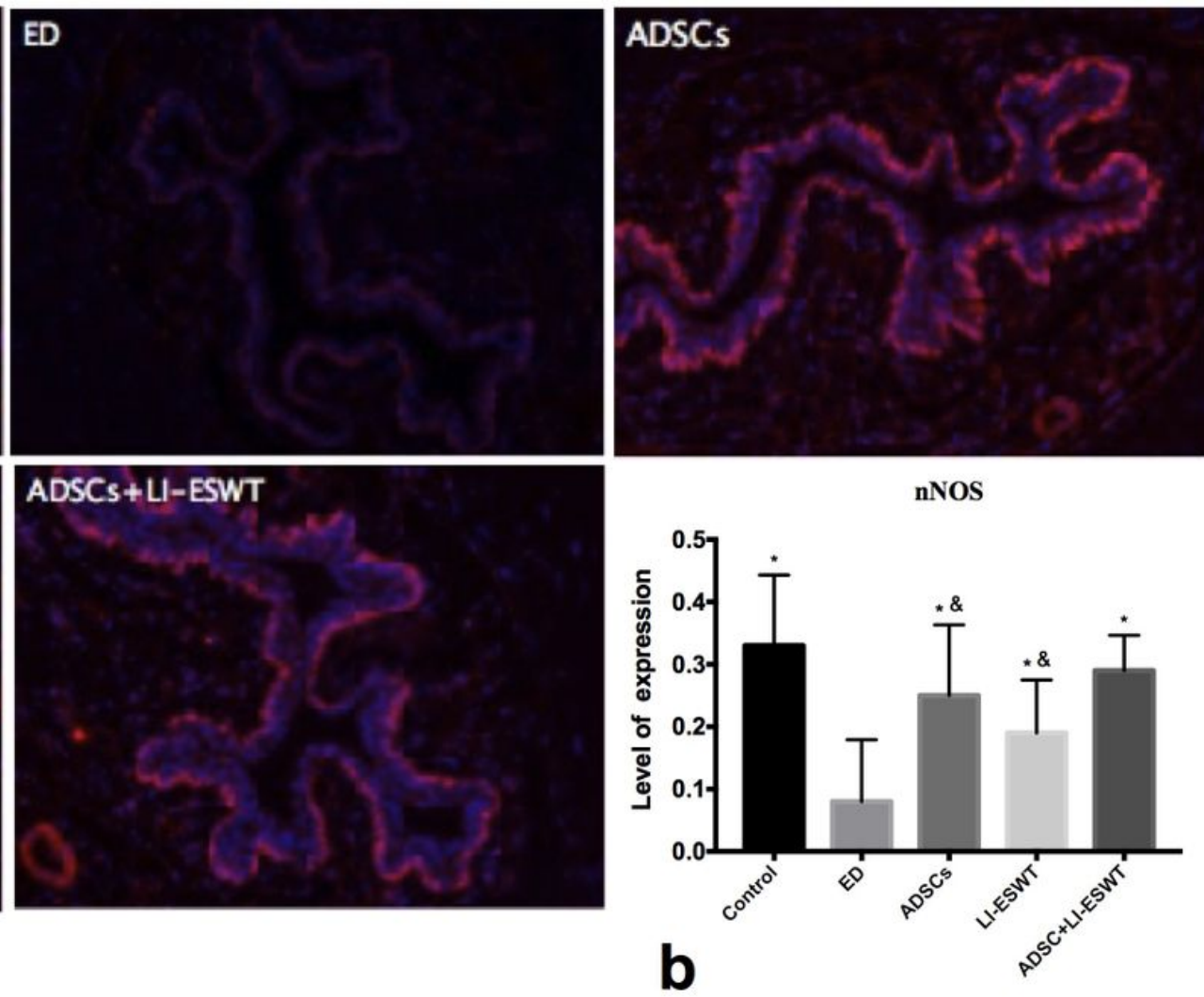

\section{Figure 6}

The nNOS expression in Corpus Cavernosum (CC). (a) Representative images of nNOS expression in control group, ED, ADSCs, LI-ESWT, ADSCs +LI-ESWT respectively, original magnification: $\times 200$; (b) Expression level of nNOS in each group, in each column and expressed as mean \pm standard deviation, $* \mathrm{P}<0.05$ when compared with ED group. \&P $<0.05$ when compared with ADSCs +LI-ESWT group

\section{Supplementary Files}

This is a list of supplementary files associated with this preprint. Click to download.

- Table2.docx

- Table3.docx

- Table1.docx

- NC3RsARRIVEGuidelinesChecklistfillable.pdf 